\title{
Dual-Tracer PET/CT Imaging in Evaluation of Metastatic Hepatocellular Carcinoma
}

\author{
Chi-lai Ho, Sirong Chen, David W.C. Yeung, and Thomas K.C. Cheng \\ Department of Nuclear Medicine and PET, Hong Kong Sanatorium and Hospital, Hong Kong, China
}

We have reported previously that ${ }^{11} \mathrm{C}$-acetate $\left({ }^{11} \mathrm{C}\right.$-ACT) PET was complementary to ${ }^{18} \mathrm{~F}-\mathrm{FDG}$ PET in the evaluation of primary hepatocellular carcinoma $(\mathrm{HCC})$ in relation to the degree of tumor cellular differentiation. In this retrospective study, our goals were to further explore the complementary role of ${ }^{11} \mathrm{C}$-ACT and ${ }^{18} \mathrm{~F}-\mathrm{FDG}$ PET in the detection of metastatic HCC disease, to evaluate the tracer characteristics of individual organ metastasis, to identify the risk factors of metastasis, and to evaluate how these results could affect patient management. Methods: One hundred twenty-one patients were selected for this study. All patients had undergone a "dual-tracer" PET/CT same-day protocol with ${ }^{11} \mathrm{C}-\mathrm{ACT}$ PET/CT followed by ${ }^{18} \mathrm{~F}-\mathrm{FDG}$ PET/CT. Sets of criteria were chosen to define "metastasis" and "no metastasis" on a patient basis. The patients considered as truepositive $(n=97)$ were then divided into 4 groups on the basis of their primary HCC tracer avidity: ${ }^{18} \mathrm{~F}-\mathrm{FDG}$-avid group, ${ }^{11} \mathrm{C}-\mathrm{ACT}$-avid group, ${ }^{18} \mathrm{~F}-\mathrm{FDG}$ - and ${ }^{11} \mathrm{C}$-ACT-avid group, and a posttreatment group with metastasis but no baseline dual-tracer PET characterization of the primary tumor and no hepatic recurrence. Results: On a patient basis, dual-tracer PET/CT had a sensitivity of $98 \%$, a specificity of $86 \%$, a positive predictive value of $97 \%$, a negative predictive value of $90 \%$, and an accuracy of $96 \%$ in the detection of HCC metastasis. On a lesion basis, 273 metastatic HCC lesions considered as true-positive were detected and categorized according to the organ or site of metastasis: lymph node (abdominal and thoracic, 49\%), lung (32\%), bone $(8 \%)$, and others (10\%). The lesion-based and patientbased detection sensitivities were $60 \%$ and $64 \%$, respectively, by ${ }^{11} \mathrm{C}-\mathrm{ACT}$ and $77 \%$ and $79 \%$, respectively, by ${ }^{18} \mathrm{~F}-\mathrm{FDG}$, and they were complementary. In analyzing lesion tracer avidity, there was a positive statistical correlation between primary HCC avidity with the general tendency of metastasis. Clinically significant changes in management were found in patients with true-positive metastasis, of whom $19 \%$ were affected by ${ }^{11} \mathrm{C}$ ACT PET alone. Dual-tracer PET/CT was more effective than single-tracer PET/CT in identifying candidates for curative therapy (negative predictive value of dual-tracer, ${ }^{18} \mathrm{~F}-\mathrm{FDG}$, and ${ }^{11} \mathrm{C}-\mathrm{ACT}$ PET/CT: $90 \%, 49 \%$, and 37\%, respectively). Conclusion: This study confirmed that ${ }^{18} \mathrm{~F}$-FDG PET/CT is useful in the evaluation of HCC metastasis, although its role in the diagnosis of primary HCC is more limited. Dual-tracer PET/CT had an incremental value and complementary advantage when compared with single-tracer imaging in the evaluation of HCC metastasis.

Received Sep. 25, 2006; revision accepted Feb. 15, 2007.

For correspondence or reprints contact: Chi-lai Ho, MD, Department of Nuclear Medicine and PET, Hong Kong Sanatorium and Hospital, 2 Village

Rd., Happy Valley, Hong Kong, China.

E-mail: garrettho@hksh.com

COPYRIGHT @ 2007 by the Society of Nuclear Medicine, Inc.
Key Words: ${ }^{11} \mathrm{C}$-acetate; ${ }^{18} \mathrm{~F}-\mathrm{FDG}$; PET/CT; hepatocellular carcinoma; metastasis

J Nucl Med 2007; 48:902-909

DOI: 10.2967/jnumed.106.036673

$\mathbf{I}$ is known that ${ }^{18} \mathrm{~F}-\mathrm{FDG}$ PET is not sensitive enough for the diagnosis of primary hepatocellular carcinoma (HCC) $(1-3)$. Although there are limited data on the role of ${ }^{18} \mathrm{~F}-$ FDG PET in the detection of HCC metastasis, these reports suggest that ${ }^{18} \mathrm{~F}$-FDG PET could be useful in the evaluation of extrahepatic metastasis, despite its limited role in the primary diagnosis of HCC (4-8).

We have reported previously that another PET tracer, ${ }^{11} \mathrm{C}$-acetate $\left({ }^{11} \mathrm{C}\right.$-ACT), was complementary to ${ }^{18} \mathrm{~F}$-FDG in the detection of primary $\mathrm{HCC}(1,9)$. The HCC tumors that were not evident with ${ }^{18} \mathrm{~F}-\mathrm{FDG}$ were detected by ${ }^{11} \mathrm{C}$-ACT. The pattern of tracer uptake by the tumors was correlated with tumor cellular differentiation. The well-differentiated HCCs preferentially accumulate ${ }^{11} \mathrm{C}$-ACT, whereas the poorly differentiated tumors tend to be ${ }^{18} \mathrm{~F}-\mathrm{FDG}$ avid. In evaluating malignant lesions in the liver, the ${ }^{11} \mathrm{C}$-ACT tracer was quite specific for $\mathrm{HCC}$, as this tracer was not accumulated in pure cholangioadenocarcinoma and metastatic liver tumors from various primary malignancies. Therefore, the combined use of ${ }^{11} \mathrm{C}-\mathrm{ACT}$ and ${ }^{18} \mathrm{~F}-$ FDG - the so-called "dual-tracer" PET or PET/CT protocolhas been used in this center since 2000 to evaluate patients with known HCC or suspicious liver masses.

In this retrospective study, we proposed the use of the same dual-tracer protocol for the evaluation of HCC metastasis. The goals of this research were to evaluate the detection sensitivity of metastatic HCC lesions with both ${ }^{11} \mathrm{C}-\mathrm{ACT}$ and ${ }^{18} \mathrm{~F}-\mathrm{FDG}$, compared with that of ${ }^{18} \mathrm{~F}-\mathrm{FDG}$ alone; to perform analysis on the dual-tracer uptake pattern of the metastatic lesions and their organs or sites of metastatic involvement; and to correlate these results with clinical outcome in terms of their usefulness in affecting subsequent patient management.

\section{MATERIALS AND METHODS}

\section{Patient Selection and Study Criteria}

We reviewed all patients referred to this center from 2002 to 2006 for dual-tracer $\left({ }^{11} \mathrm{C}-\mathrm{ACT}\right.$ and $\left.{ }^{18} \mathrm{~F}-\mathrm{FDG}\right) \mathrm{PET} / \mathrm{CT}$ 
evaluation of liver masses. Only those patients with confirmed primary HCC malignancy (by histopathology or by Barcelona criteria (10)) and no other clinical suspicion of second cancers were included. On the basis of the availability and reliability of follow-up information, 121 patients (96 men, 25 women; age range, $16-81 \mathrm{y}$; mean age, $58.6 \pm 12.7$ y [mean $\pm \mathrm{SD}])$ were included in the study population. Eighty-one patients were positive for hepatitis B surface antigen, and 4 patients were positive for hepatitis $\mathrm{C}$.

Confirmation of Metastasis. Because histopathologic confirmation of metastasis was not possible in all patients or in all lesions, a set of criteria was designed to include other clinical, biochemical, and imaging parameters as objective evidence that a lesion detected by PET/CT was most likely a metastasis. A lesion satisfying 1 or more of the following criteria was considered a metastasis: (a) histopathologic confirmation of metastasis by resection or biopsy, (b) biochemical evidence of increasing $\alpha$-fetoprotein concentration and clinical follow-up, (c) 2 or more serial PET/CT studies in a 3- to 7-mo period with unequivocal evidence of progression, and (d) additional or follow-up radiologic evidence of bone and lung metastases (evidence of bone destruction, increase in number and size of satellite lung nodules with no clinical evidence of infection).

Confirmation of No Metastasis. A patient satisfying all the following criteria was considered to be without metastasis: (a) diagnosis as having no extrahepatic or intravascular metastasis by pretreatment dual-tracer PET/CT, (b) primary HCC tumors completely resected, and (c) follow-up PET/CT and other diagnostic studies showing no extrahepatic metastasis in a 5- to 18-mo follow-up interval (median, $11 \mathrm{mo}$ ).

The true-positive (TP) patients were then divided into 4 groups on the basis of their primary HCC tracer avidity: group I, ${ }^{18} \mathrm{~F}-\mathrm{FDG}$ avid; group II, ${ }^{11} \mathrm{C}$-ACT avid; group III, ${ }^{18} \mathrm{~F}$-FDG and ${ }^{11} \mathrm{C}$-ACT avid; and group IV, patients with extrahepatic metastasis in whom the primary tumor tracer avidity was not evaluable because their primary HCC was resected or ablated before PET/CT evaluation and no hepatic recurrence was found.

Criteria Used to Determine a Change in Management. The patients referred for initial staging were originally scheduled for curative resection if PET/CT was negative for metastasis. Therefore, for these patients, any positive evidence of metastasis found by PET/CT was considered to lead to a change in management if the patient did not go for surgery or ablation of the primary HCC or the patient underwent resection or ablation of both the primary tumor and metastatic foci. For patients who were referred for restaging, a positive PET/CT finding that led to further treatment was considered a change in management. For patients who were initially judged as having metastasis by radiologic findings, a negative PET/CT finding, which convinced the surgeons to undertake surgery, that confirmed no metastasis was also considered a change in management.

This study was approved by the Ethics and Research Committee of the authors' clinical institution for investigation of primary $\mathrm{HCC}$, and all patients gave informed and written consent before the imaging procedure was undertaken. Analysis of the data for metastatic HCC was subsequently retrospective. The patients had fasted for at least $6 \mathrm{~h}$ and the blood glucose concentration was determined before injection of PET radiopharmaceuticals. All patients except 2 had glucose concentrations below $7 \mathrm{mmol} / \mathrm{L}$. Both hyperglycemic patients (with blood glucose of 8.1 and 8.5 $\mathrm{mmol} / \mathrm{L}$ ) were given 2 units of short-acting insulin intravenously and waited for $1 \mathrm{~h}$ after confirmation of normoglycemic status before injection of PET radiopharmaceuticals.

\section{Dual-Tracer PET/CT}

${ }^{11} \mathrm{C}$-ACT was prepared by modifying the methodology and setup as reported by Norenberg (11). ${ }^{11} \mathrm{C}-\mathrm{ACT}$ (550-740 MBq) was administered intravenously, and imaging of the whole-body was performed at $20 \mathrm{~min}$ after injection using an integrated in-line PET/CT scanner (Biograph lutetium oxyorthosilicate [LSO] or Biograph 16 LSO HI-REZ; Siemens Medical Solutions USA, Inc.). Data acquisition began with CT (with no contrast agent) at $130 \mathrm{kVp}, 110-115 \mathrm{~mA}, 2-\mathrm{mm}$ pitch, and 1-s tube rotation; this was followed by PET with a 2-min emission acquisition time of 47 (Biograph) or 81 (Biograph 16) axial image planes simultaneously at a $16.2-\mathrm{cm}$ axial field of view per position. The images were reconstructed by means of the standardized ordered-subset expectation maximization (OSEM) technique using 8 subsets and 2 iterations with a $128 \times 128$ matrix $($ Biograph) or $168 \times 168$ matrix (Biograph 16) for PET and a $512 \times 512$ matrix for CT. The average reconstructed $x-y$ spatial resolution for PET was about $3.5-\mathrm{mm}$ full width at half maximum in-plane (at $1 \mathrm{~cm}$ ).

About $15 \mathrm{~min}$ after ${ }^{11} \mathrm{C}$-ACT imaging, ${ }^{18} \mathrm{~F}-\mathrm{FDG}$ (370-550 $\mathrm{MBq}$ ) was injected intravenously (about $45 \mathrm{~min}$ after initial ${ }^{11} \mathrm{C}$ ACT injection). Scanning with the same imaging positions and acquisition settings of the whole-body or upper abdomen began at 60 min after ${ }^{18} \mathrm{~F}$-FDG administration. This allowed a total of about 105 min after initial injection of ${ }^{11} \mathrm{C}$-ACT, $>5$ decay half-lives of ${ }^{11} \mathrm{C}$ (20 min). Attenuation correction, reconstruction parameters, and semiquantitative analysis were similar to the specifications as stated earlier.

\section{Interpretation Criteria and Statistical Analysis}

A lesion was regarded as positive for metastasis on the basis of visual judgment of the degree of increased metabolism by 3 experienced and independent interpreters, supported by semiquantitative evaluation based on calculation of the standardized uptake value (SUV) in both sets of ${ }^{18} \mathrm{~F}$-FDG and ${ }^{11} \mathrm{C}$-ACT PET. Both maximum (SUVmax) and average (SUVavg) SUVs were calculated. A SUVmax $>2.0$ was generally used as the semiquantitative criterion of metastasis in both ${ }^{18} \mathrm{~F}-\mathrm{FDG}$ and ${ }^{11} \mathrm{C}$-ACT PET. The McNemar test was adopted to compare the avidity difference between ${ }^{18} \mathrm{~F}$-FDG and ${ }^{11} \mathrm{C}$-ACT PET/CT. Group comparisons of tracer avidity differences were tested for significance using the $\chi^{2}$ test. If serial PET/CT studies persistently did not show increased metabolism and size by either tracer, the lesions seen on CT (particularly enlarged abdominal lymph nodes) were regarded as negative for metastasis. If $>3$ metastatic lesions of lung or bone were seen, these were counted as 3 lesions maximum. This was done to avoid bias of statistical counting by one or a few patients presenting with an advanced stage of metastasis with numerous metastatic lung or bone lesions. Metastatic lymph nodes were counted in accordance with location. Peritoneal and omental lesions were classified as soft-tissue metastasis because of their small number and for simplicity of data analysis.

A "TP" case was defined as follows: Single- or dual-tracer PET/CT correctly detects at least 1 metastasis (regardless of the accuracy of the other metastatic lesions) in a patient who truly has metastasis and is thus not a candidate for curative therapy. A "true-negative" (TN) case was defined by the usual convention that single- or dual-tracer PET/CT correctly identifies a patient who truly had no metastasis. 


\section{RESULTS}

\section{Patient-Based Analysis}

Among the 121 patients, 21 were diagnosed as having no metastasis by dual-tracer PET/CT. The follow-up period of this control group was 5-18 mo (median, 11 mo). During this period, 19 patients who satisfied the preset criteria of having no metastasis remained well by clinical, biochemical, and follow-up PET/CT. One FN patient had multiple metastatic mediastinal nodes that initially showed very minimal to no ${ }^{11} \mathrm{C}$-ACT and ${ }^{18}$ F-FDG activity on preoperative PET/CT. After 2 serial imaging studies (5 mo later), most of the mediastinal nodes began to show increased ${ }^{11} \mathrm{C}$-ACT metabolism and enlargement with progressively rising $\alpha$-fetoprotein concentration but without primary tumor recurrence in liver. The other FN patient had a markedly elevated $\alpha$-fetoprotein concentration and multiple ${ }^{18} \mathrm{~F}-\mathrm{FDG}$ - and ${ }^{11} \mathrm{C}$-ACT-avid lung nodules on follow-up PET/CT 7 mo after primary HCC resection. Retrospective review of the baseline PET/CT showed that 2 subcentimeter lung nodules could have been faintly present on $\mathrm{CT}$ but had no abnormal ${ }^{18} \mathrm{~F}-\mathrm{FDG}$ or ${ }^{11} \mathrm{C}$-ACT uptake.

One hundred patients diagnosed by dual-tracer PET/CT had metastasis. Among them, 97 patients satisfied the preset criteria of metastasis. The follow-up period of serial PET/CT was 3-7 mo (median, 4 mo). The 3 falsepositive (FP) cases with 7 lung lesions $\leq 1 \mathrm{~cm}$ were faintly seen on CT. They were all mildly ${ }^{18}$ F-FDG avid and either resolved on subsequent follow-up PET/CT or remained static without increased serum $\alpha$-fetoprotein levels.

On a patient basis, dual-tracer PET/CT had a sensitivity of $98 \%$ (97/99), a specificity of $86 \%$ (19/22), a positive predictive value (PPV) of 97\% (97/100), a negative predicative value (NPV) of $90 \%(19 / 21)$, and an accuracy of $96 \%(116 / 121)$ in the detection of HCC metastasis (Table 1). The corresponding data for single-tracer PET/CT (based on the criteria defined earlier) are also summarized in Table 1. The patient-based sensitivities of ${ }^{18} \mathrm{~F}-\mathrm{FDG}$ and ${ }^{11} \mathrm{C}$-ACT PET/CT were $79 \%$ and $64 \%$, respectively. However, the NPV was $<50 \%$ when using either tracer alone. This implies that negative single-tracer PET/CT may not be sufficient to choose the correct candidate for curative therapy in view of the high $\mathrm{FN}$ rate in the detection of $\mathrm{HCC}$ metastasis.

\section{Grouping of Patients According to Primary HCC Tracer Avidity}

Ninety-seven patients satisfied the preset criteria for HCC metastasis, with 273 metastatic HCC lesions found in these patients. The patients were categorized into 4 groups on the basis of the tracer avidity of their primary HCC tumors. Group I consisted of 13 patients with primary HCC tumors avid for ${ }^{18} \mathrm{~F}-\mathrm{FDG}$ only, group II had 15 patients with primary $\mathrm{HCC}$ tumors avid for ${ }^{11} \mathrm{C}$-ACT only, and group III had 47 patients with primary HCC tumors avid for both ${ }^{18} \mathrm{~F}$-FDG and ${ }^{11} \mathrm{C}$-ACT. Group IV consisted of 22 patients who had no primary HCC recurrence in liver after previous treatment (partial hepatectomy, radiofrequency ablation, or liver transplantation) for $\mathrm{HCC}$ without baseline dual-tracer PET but were found to have extrahepatic HCC metastasis by dual-tracer PET/CT.

\section{Detection Analysis According to Metastatic Lesion Tracer Avidity}

Of these 273 metastatic HCC lesions, 18 were intravascular (portal and venous) and 255 were extrahepatic. The percentages of total lesions avid for ${ }^{11} \mathrm{C}-\mathrm{ACT},{ }^{18} \mathrm{~F}-\mathrm{FDG}$, and both tracers were $23 \%(n=62), 40 \%(n=110)$, and $37 \%$ ( $n=101)$, respectively. Therefore, the lesion-based sensitivities of ${ }^{11} \mathrm{C}-\mathrm{ACT}$ and ${ }^{18} \mathrm{~F}-\mathrm{FDG}$ in the detection of metastatic HCC were $60 \%$ and $77 \%$, respectively.

\section{Detection Analysis According to Site of Metastasis}

In our study, the most frequent site of metastasis was lymph nodes, $49 \%$ of the total 273 metastatic lesions $(n=$ 135; 63 thoracic nodes and 72 abdominal nodes; size range, 0.6-6.7 cm; mean size, $1.97 \pm 0.97 \mathrm{~cm}$ ); this was followed by lung, $32 \%$ ( $n=88$; size range, $0.6-6.0 \mathrm{~cm}$; mean size, $1.82 \pm 0.96 \mathrm{~cm})$, bone, $8 \%(n=23$; size range, $1.0-6.2$ $\mathrm{cm}$; mean size, $3.25 \pm 1.53 \mathrm{~cm})$, vascular, $7 \%(n=18)$, adrenal gland, $2 \%(n=6)$, and soft tissue, $1 \%(n=3)$. Table 2 summarizes the SUVmax statistics of the metastatic HCC lesions in lymph node, lung, and bone by dual-tracer PET/CT. To compare these data with the data in the literature, the results were calculated again on the basis of the number of patients having individual organ metastasis. On a patient basis, metastasis was as follows: lung, 43\%; abdominal node, 34\%; thoracic node, $25 \%$; bone, $15 \%$; vascular, 16\%; adrenal gland, 6\%; and soft tissue, $3 \%$. There were 139 metastatic sites, averaging 1.43 sites per patient.

TABLE 1

Patient-Based Statistics of Diagnostic Values of Single-Tracer and Dual-Tracer PET/CT

\begin{tabular}{cccccc}
\hline Tracer & Sensitivity (\%) & Specificity (\%) & PPV (\%) & NPV (\%) & Accuracy (\%) \\
\hline${ }^{18}$ F-FDG* $^{*}$ ( $^{*}$ & $79(78 / 99)$ & $91(20 / 22)$ & $98(78 / 80)$ & $49(20 / 41)$ & $81(98 / 121)$ \\
${ }^{*}$ Dual-tracer & $64(63 / 99)$ & $95(21 / 22)$ & $98(63 / 64)$ & $37(21 / 57)$ & $69(84 / 121)$ \\
& $98(97 / 99)$ & $86(19 / 22)$ & $97(97 / 100)$ & $90(19 / 21)$ & $96(116 / 121)$
\end{tabular}

${ }^{*}$ Definition of TP for single-tracer PET/CT: correct detection of at least 1 metastatic lesion in a patient with metastatic HCC disease. 
TABLE 2

SUVmax of HCC Lesions Considered to be True-Positive

\begin{tabular}{lcc}
\hline \multicolumn{1}{c}{ Lesion site } & ${ }^{18} \mathrm{~F}-\mathrm{FDG}$ & ${ }^{11} \mathrm{C}-\mathrm{ACT}$ \\
\hline Lymph nodes $(n=135)$ & $5.06 \pm 2.95$ & $5.44 \pm 2.37$ \\
Lung $(n=88)$ & $3.99 \pm 2.36$ & $3.71 \pm 1.73$ \\
Bone $(n=23)$ & $4.75 \pm 2.22$ & $7.29 \pm 5.09$ \\
& & \\
\multicolumn{2}{l}{ Values are expressed as mean \pm SD. } \\
\hline
\end{tabular}

\section{Detection Analysis According to Primary HCC Tracer Avidity}

Of the 110 metastatic lesions detected by ${ }^{18} \mathrm{~F}-\mathrm{FDG}, 62$ lesions detected by ${ }^{11} \mathrm{C}-\mathrm{ACT}$, and 101 lesions detected by both tracers, each category of these metastatic lesions was further classified according to the grouping method as indicated earlier (according to tracer avidity of their primary HCC tumors). The results are summarized in Table 3.

In group I, there were 41 metastatic lesions. Of these 41 lesions (6 lesions avid for both tracers), ${ }^{18} \mathrm{~F}-\mathrm{FDG}$ detected $36(88 \%)$ and ${ }^{11} \mathrm{C}$-ACT detected $11(27 \%)(P \ll 0.05$, McNemar test). The $12 \%$ metastatic lesions that were negative on ${ }^{18} \mathrm{~F}-\mathrm{FDG}$ were detected by ${ }^{11} \mathrm{C}$-ACT. In this group of patients, the most frequent site of metastasis was the lymph nodes (27/41 lesions, $66 \%)$.

In group II, there were 43 metastatic lesions. Of these 43 lesions (12 lesions avid for both tracers), ${ }^{18} \mathrm{~F}-\mathrm{FDG}$ detected $29(67 \%)$ and ${ }^{11} \mathrm{C}$-ACT detected $26(60 \%)(P \gg 0.05$, McNemar test). This implied that the use of either tracer alone would have a 30\%-40\% FN detection rate of metastasis in this group of patients. However, these 2 tracers were complementary to each other as in group I, with all of the metastatic lesions detected by the combination of both tracers. This group of patients had the highest incidence of detection in lung-pleura metastasis (25/43 lesions, 58\%).

In group III, there were 122 metastatic lesions. With 44 lesions avid for both tracers, ${ }^{18} \mathrm{~F}-\mathrm{FDG}$ detected 95 lesions (78\%) and ${ }^{11} \mathrm{C}$-ACT detected 71 lesions $(58 \%)(P \ll 0.05$, McNemar test). Lymph node metastasis (thoracic plus abdominal) had the highest incidence in this group of patients (68/122 lesions, 56\%).

In group IV, the group without primary HCC recurrence, there were 67 metastatic lesions (39 lesions avid for both tracers). ${ }^{18} \mathrm{~F}-\mathrm{FDG}$ detected $51(76 \%)$ and ${ }^{11} \mathrm{C}$-ACT detected $55(82 \%)$. Although the difference was not statistically significant $(P \gg 0.05$, McNemar test), this was the only group of patients with the detection sensitivity higher using ${ }^{11} \mathrm{C}$-ACT than that using ${ }^{18} \mathrm{~F}-\mathrm{FDG}$. Statistically, it was also significantly higher than the ${ }^{11} \mathrm{C}$-ACT detection sensitivity in the other 3 groups $(P \ll 0.05$ for group I, $P \ll 0.05$ for group II, $P \ll 0.05$ for group III; $\chi^{2}$ test). Lung-pleura was the organ or site with the highest rate of metastasis (32/67 lesions, 48\%). Of these 32 lesions, ${ }^{11} \mathrm{C}$-ACT detected 29 $(91 \%)$ and ${ }^{18} \mathrm{~F}-\mathrm{FDG}$ detected $18(56 \%)(P \ll 0.05$,
TABLE 3

Tracer Avidity Statistics of Number of HCC Metastases in Groups I-IV

\begin{tabular}{|c|c|c|c|c|}
\hline \multicolumn{5}{|c|}{ Group I } \\
\hline Organ of metastasis & ${ }^{18} \mathrm{~F}-\mathrm{FDG}$ & ${ }^{11} \mathrm{C}-\mathrm{ACT}$ & Both & Total \\
\hline Lymph node & 20 & 4 & 3 & 27 \\
\hline Lung & 4 & 1 & 1 & 6 \\
\hline Bone & 2 & 0 & 2 & 4 \\
\hline Vascular & 3 & 0 & 0 & 3 \\
\hline Adrenal gland & 1 & 0 & 0 & 1 \\
\hline Soft tissue & 0 & 0 & 0 & 0 \\
\hline Total & 30 & 5 & 6 & 41 \\
\hline \multicolumn{5}{|c|}{ Group II } \\
\hline Organ of metastasis & ${ }^{18} \mathrm{~F}-\mathrm{FDG}$ & ${ }^{11} \mathrm{C}-\mathrm{ACT}$ & Both & Total \\
\hline Lymph node & 5 & 7 & 2 & 14 \\
\hline Lung & 12 & 4 & 9 & 25 \\
\hline Bone & 0 & 1 & 1 & 2 \\
\hline Vascular & 0 & 1 & 0 & 1 \\
\hline Adrenal gland & 0 & 0 & 0 & 0 \\
\hline Soft tissue & 0 & 1 & 0 & 1 \\
\hline Total & 17 & 14 & 12 & 43 \\
\hline \multicolumn{5}{|c|}{ Group III } \\
\hline Organ of metastasis & ${ }^{18} \mathrm{~F}-\mathrm{FDG}$ & ${ }^{11} \mathrm{C}-\mathrm{ACT}$ & Both & Total \\
\hline Lymph node & 28 & 18 & 22 & 68 \\
\hline Lung & 13 & 3 & 9 & 25 \\
\hline Bone & 3 & 3 & 6 & 12 \\
\hline Vascular & 6 & 2 & 6 & 14 \\
\hline Adrenal gland & 1 & 0 & 0 & 1 \\
\hline Soft tissue & 0 & 1 & 1 & 2 \\
\hline Total & 51 & 27 & 44 & 122 \\
\hline \multicolumn{5}{|c|}{ Group IV } \\
\hline Organ of metastasis & ${ }^{18} \mathrm{~F}-\mathrm{FDG}$ & ${ }^{11} \mathrm{C}-\mathrm{ACT}$ & Both & Total \\
\hline Lymph node & 6 & 1 & 19 & 26 \\
\hline Lung & 3 & 14 & 15 & 32 \\
\hline Bone & 2 & 0 & 3 & 5 \\
\hline Vascular & 0 & 0 & 0 & 0 \\
\hline Adrenal gland & 1 & 1 & 2 & 4 \\
\hline Soft tissue & 0 & 0 & 0 & 0 \\
\hline Total & 12 & 16 & 39 & 67 \\
\hline
\end{tabular}

McNemar test), indicating that ${ }^{11} \mathrm{C}-\mathrm{ACT}$ is the more important tracer in this group of patients in the detection of lung metastasis.

A summary of the individual tracer detection sensitivities for all 4 groups of patients is given in Table 4 .

\section{Results of Individual Organ Metastasis According to Tracer Avidity}

Further breakdown of the organ metastasis according to tracer avidity is also summarized in Table 3, which shows that ${ }^{18} \mathrm{~F}-\mathrm{FDG}$ was better than or equal to ${ }^{11} \mathrm{C}-\mathrm{ACT}$ in identifying metastatic lesions in each of the organ categories. ${ }^{18} \mathrm{~F}-\mathrm{FDG}$ alone detected $78 \%(105 / 135)$ of the metastatic lymph nodes, $75 \%$ (66/88) of lung-pleura 
TABLE 4

Sensitivity of ${ }^{11} \mathrm{C}-\mathrm{ACT}$ and ${ }^{18} \mathrm{~F}-\mathrm{FDG} \mathrm{PET} / \mathrm{CT}$ in Detection of HCC Metastasis

\begin{tabular}{lll}
\hline & \multicolumn{2}{c}{ Sensitivity (\%) } \\
\cline { 2 - 3 } Group & \multicolumn{1}{c}{${ }^{18}$ F-FDG } & $27(11 / 41)$ \\
\hline I & $88(36 / 41)$ & $60(26 / 43)$ \\
II & $67(29 / 43)$ & $58(71 / 122)$ \\
III & $78(95 / 122)$ & $82(55 / 67)$ \\
IV & $76(51 / 67)$ & \\
\hline
\end{tabular}

lesions, $83 \%(19 / 23)$ of bone metastases, $83 \%(15 / 18)$ of vascular metastases, and 67\% (6/9) of adrenal and soft-tissue metastases. ${ }^{11} \mathrm{C}$-ACT alone detected $56 \%$ (76/ $135)$ of the metastatic lymph nodes, $64 \%$ (56/88) of lungpleura lesions, $70 \%(16 / 23)$ of bone metastases, $50 \%(9 / 18)$ of vascular metastases, and $67 \%$ (6/9) of adrenal and softtissue metastases. These data showed that the complementary relationship between these 2 tracers was also found in the detection of metastasis similar to that noted previously in the primary $\mathrm{HCC}(1,9)$.

\section{Change in Patient Management}

Of the 97 patients with metastasis, 47 patients (48\%) had evaluation during their first presentations and were not known to have metastasis before imaging. Figure 1 summarizes the change in management as a result of the dualtracer PET/CT findings. After PET/CT, 36 patients chose to have no further treatment or some form of alternative treatment. They all died after a median survival of $<1 \mathrm{y}$ and avoided unnecessary surgery that would not have lengthened their survival. Eight patients chose to undergo chemotherapy or focused radiation therapy. One patient was successfully down-staged on the basis of serial followup PET/CT and subsequently underwent resection of her primary HCC tumor. She was alive at 18 mo of follow-up. Three of the 47 patients had their primary and secondary tumors (single-organ metastasis) resected in one operation.

Similarly, the remaining 50 patients with previous treatments who were found to have metastasis by dual-tracer PET/ CT were also given different forms of treatment or no treatment on the basis of the PET/CT findings. Twenty-three patients died within $1 \mathrm{y}$ without treatment, 18 patients had chemotherapy or radiation therapy ( 2 of whom were downstaged for surgery on the basis of the serial follow-up PET/ CT), and 9 patients had surgical resection of metastasis (Fig. 1).

Of the $19 \mathrm{TN}$ patients, 3 with cirrhosis had abdominal lymphadenopathy initially diagnosed as metastasis by CT/ MRI. Benign enlargement of abdominal nodes is frequently found in cirrhotic patients (12). These 3 patients underwent curative resection after dual-tracer PET/CT revealed negative findings. The lymph nodes were confirmed as negative for metastasis either intraoperatively or by histopathologic examination, and follow-up over 5-18 mo showed no evidence for extrahepatic metastasis.

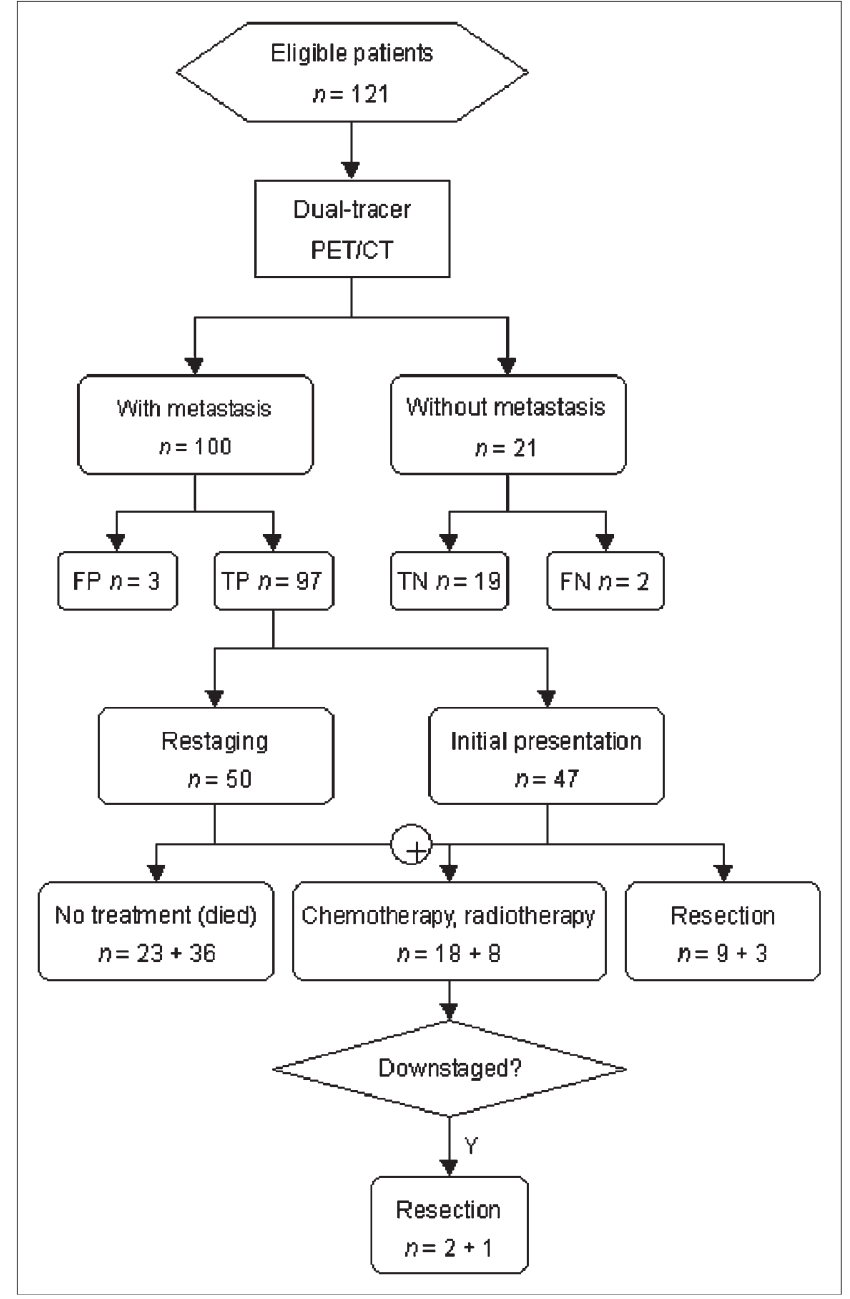

FIGURE 1. Flow chart of patient distribution shows 2 arms of analysis according to PET/CT diagnosis of HCC metastasis. Of 19 TN patients, 10 underwent curative resection and 9 were treated by radiofrequency ablation. Two FN patients initially underwent resection of their primary HCCs and, during followup of approximately 5-7 mo, dual-tracer PET/CT revealed metastasis, which was further treated by chemotherapy. $Y=$ yes.

\section{DISCUSSION}

The nature and characteristics of HCC metastasis have not been described in detail in the literature. Presumably, one of the major reasons is that this is not a common type of cancer in the Euro-American population. Furthermore, it has been suggested that conventional methods of imaging are somewhat insensitive for staging HCC (13). The existing data on conventional and nonconventional methods for evaluation of HCC metastasis are scarce in the literature. CT is the standard tool for conventional imaging (14), which is based primarily on the assumption that most HCC tumors-primary and metastatic-are hypervascular, and the arterial phase should demonstrate increased contrast enhancement within the lesion. With today's multidetector and high-resolution technology, CT is particularly useful for evaluation of small lesions 
and determination of the spatial relationship between the tumor and the background normal tissue architecture. However, the increased sensitivity may likely result in overdiagnosis of some other hypervascular tumor or tumor-like lesions, particularly when they are small in size (15). On the other hand, differentiation between recurrence and posttreatment scarring changes and between metastasis and reactive lymph node enlargement are less effective using CT alone. Detection of osseous metastatic disease is another known limitation of CT, as most metastatic bone lesions from HCC are osteolytic $(16,17)$ and are evident on CT only as a late manifestation.

In recent years, ${ }^{18} \mathrm{~F}-\mathrm{FDG}$ PET and PET/CT are increasingly important tools for tumor staging. Despite the fact that ${ }^{18}$ F-FDG PET has only a fair sensitivity in diagnosis of the primary HCC tumor, Sugiyama et al. (6) and Bohm et al. (4) have reported that ${ }^{18}$ F-FDG PET may have incremental value in detection of HCC secondary tumors. The detection sensitivities were $83 \%$ and $64 \%$, respectively. Chen et al. (5) showed that ${ }^{18}$ F-FDG PET identified the source of elevated $\alpha$-fetoprotein in $73 \%$ of their patients after treatment of HCC. Wudel and Delbeke's group (8) also showed that ${ }^{18} \mathrm{~F}-\mathrm{FDG}$ PET affected management in $28 \%$ of a group of 91 HCC patients, including a subgroup with extrahepatic metastasis. In our study, using ${ }^{18} \mathrm{~F}-\mathrm{FDG}$ alone, PET/CT had a patient-based sensitivity of $79 \%$ and a lesion-based sensitivity of $77 \%$. These sensitivities were closer to the value of Sugiyama et al. than those of Bohm et al. However, when the lesion-based sensitivity was further categorized in individual groups (Table 4), ${ }^{18} \mathrm{~F}-\mathrm{FDG}$ had the highest sensitivity $(88 \%)$ in the detection of HCC metastasis when the primary HCC tumor was also ${ }^{18} \mathrm{~F}-\mathrm{FDG}$ avid. On the other hand, when the primary HCC was ${ }^{11} \mathrm{C}$-ACT avid, ${ }^{18} \mathrm{~F}-\mathrm{FDG}$ had the lowest sensitivity $(67 \%)$. Thus, the sensitivity of ${ }^{18}$ F-FDG PET in detecting metastatic lesions is dependent on the avidity of the primary tumor tracer, which reflects the degree of cellular differentiation of the primary HCC tumor. These 2 values are individually comparable with those reported by Sugiyama et al. and Bohm et al., but whether the difference had any relation to the differentiation of the primary $\mathrm{HCC}$ is uncertain.

Our study is different from those of Sugiyama et al. (6) and Bohm et al. (4) in the use of 2 PET tracers instead of one. In our 4 groups of patients, the relative $\mathrm{FN}$ rate of ${ }^{18} \mathrm{~F}$ FDG PET was 12\%-33\%; however, the undetected lesions could be detected by ${ }^{11} \mathrm{C}$-ACT PET. In other words, the complementary nature of ${ }^{11} \mathrm{C}$-ACT and ${ }^{18} \mathrm{~F}-\mathrm{FDG}$ is evident not just in primary $\mathrm{HCC}$ tumors $(1,9)$ but also in metastatic HCC lesions. Furthermore, patient management decisions were largely based on the dual-tracer PET/CT findings in our institution. The incremental value of ${ }^{11} \mathrm{C}$-ACT PET relative to that of ${ }^{18} \mathrm{~F}-\mathrm{FDG}$ PET alone is difficult to quantify, as the patients with metastatic HCC are a heterogeneous group of patients often with multiorgan and multifocal involvement. Among the 97 patients, 18 patients
(19\%) had single- or multiorgan metastases avid for ${ }^{11} \mathrm{C}$ ACT only. On a lesion basis, $23 \%$ of metastatic lesions were detected by ${ }^{11} \mathrm{C}$-ACT only. These results suggest that the incremental value of ${ }^{11} \mathrm{C}$-ACT PET in affecting patient management is significant. Individually, ${ }^{11} \mathrm{C}$-ACT PET may have greater impact on the group II and group IV patients, particularly in those cases with single-organ metastasis.

The NPV of PET/CT with either single tracer was $<50 \%$ on a patient basis (Table 1). This implies that a negative single-tracer study cannot reliably exclude metastatic HCC and, therefore, single-tracer PET/CT is not an accurate tool in identifying candidates for curative therapy. However, with dual-tracer PET/CT, the NPV increased significantly to $90 \%$ (19/21). The FN cases with dual-tracer PET/CT were patients with tiny lung lesions and mediastinal nodes of $<1 \mathrm{~cm}$ with either equivocal or minimally increased uptake of either tracer. This is a known technical limitation of PET/CT, related to partial-volume effects and respiratory motion.

\section{Primary HCC Features Affecting Likelihood of Metastasis}

From the results in this study and 2 earlier studies $(1,9)$, we have identified 3 separate groups of HCC patients who have (a) a small primary tumor with a size of $1.4 \pm 0.3 \mathrm{~cm}$ and no metastasis, (b) an intermediate primary tumor with a size of $3.5 \pm 1.9 \mathrm{~cm}$ and no metastasis, and (c) HCC with metastasis (this study). As summarized in Table 5, there is a steady increase in the percentage of primary tumors that are avid for ${ }^{18} \mathrm{~F}$-FDG, from $32 \%$ to $80 \%$, whereas the percentage for ${ }^{11} \mathrm{C}$-ACT remained stable at $83 \%-87 \%$. Because individual tracer avidity was related to cellular differentiation in HCC, one could speculate that it is more likely for metastasis to occur in cases of primary HCC tumors with poor differentiation than for those with well-differentiated pathology.

Another group of patients of special interest is group IV, where no recurrence was found in the liver previously treated for primary HCC but where there were extrahepatic metastases. This group of patients had the highest metastasis detection sensitivity $(82 \%)$ by ${ }^{11} \mathrm{C}$-ACT, quite similar to that of the primary HCC tumors of this study ( $83 \%$ by ${ }^{11} \mathrm{C}$-ACT) and the 2 earlier studies $\left(87 \%\right.$ by $\left.{ }^{11} \mathrm{C}-\mathrm{ACT}\right)$ $(1,9)$. Therefore, unlike the other 3 groups, ${ }^{11} \mathrm{C}$-ACT PET is the more important tracer in this group of patients for the detection of metastasis.

TABLE 5

Comparison of ${ }^{18} \mathrm{~F}-\mathrm{FDG}$ and ${ }^{11} \mathrm{C}-\mathrm{ACT}$ in Detection Sensitivities of Primary HCC Tumor

\begin{tabular}{lcc}
\hline \multicolumn{1}{c}{ Primary HCC group } & ${ }^{18} \mathrm{~F}-\mathrm{FDG}(\%)$ & ${ }^{11} \mathrm{C}-\mathrm{ACT}(\%)$ \\
\hline Small (no metastasis) & $32(12 / 38)$ & $87(33 / 38)$ \\
Intermediate (no metastasis) & $47(26 / 55)$ & $87(48 / 55)$ \\
HCC with metastasis & $80(60 / 75)$ & $83(62 / 75)$ \\
\hline
\end{tabular}



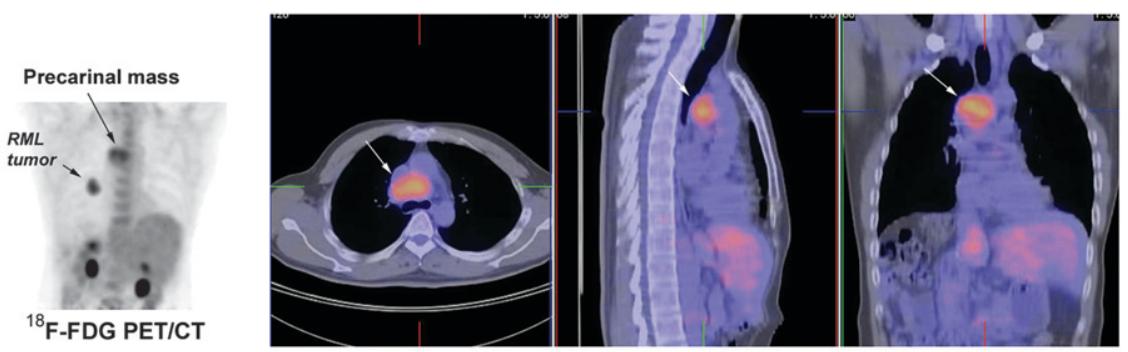

FIGURE 2. Mediastinal metastasis: a 49-y-old patient who had right hemihepatectomy for HCC 3 y earlier. Follow-up chest radiographs showed a right middle lobe (RML) mass. ${ }^{18} \mathrm{~F}-\mathrm{FDG}$ and ${ }^{11} \mathrm{C}-\mathrm{ACT}$ PET/CT showed hypermetabolic RML lung mass and large precarinal node (arrows). Biopsy confirmed metastatic HCC pathology in both locations.
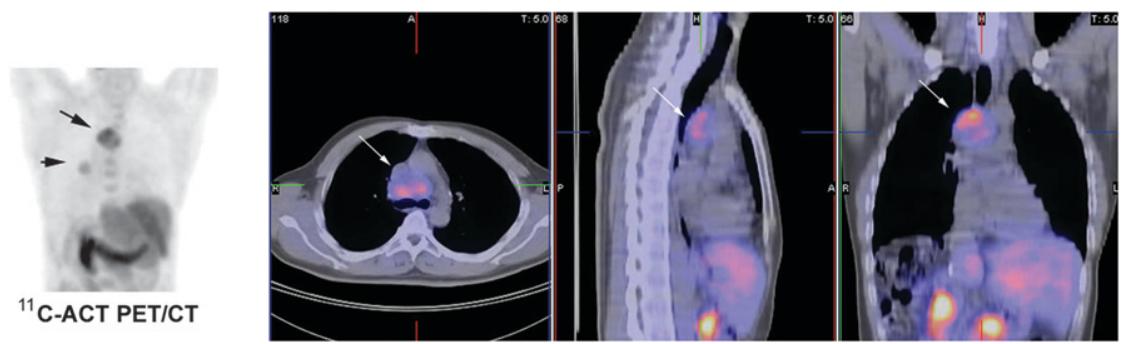

\section{Individual Organ Metastasis}

Our data show that lung metastases constitute the largest group of metastatic lesions, followed by metastases to abdominal lymph nodes, thoracic lymph nodes, bone, and other sites in descending order of prevalence. Excluding the thoracic nodes, these results are similar to those found in the literature $(14,18)$. However, our data also suggest that thoracic nodal involvement constitutes a significant site of organ metastasis (63 thoracic nodes, 72 abdominal nodes). Lymphatic spread from the liver, as one of the upper gastrointestinal malignancies, follows a specific pathway of drainage through the lesser omentum to the retroperito- neal space, to the hepatoduodenal, peripancreatic, and aortocaval nodes (19). Lymphatic spread may also ascend within the retroperitoneal space along the aortocaval chain to the subphrenic and thoracic nodal basins. As a result, the metastatic thoracic nodes that have been observed in this study include the paravertebral, paraesophageal, subcarinal, and precarinal nodes and other less common sites in the mediastinum. When these nodes are ${ }^{11} \mathrm{C}-\mathrm{ACT}$ avid, it is more likely that they truly harbor HCC metastasis. However, because biopsy of all suggestive lesions was not always possible, a small number of these intrathoracic nodes with increased ${ }^{18}$ F-FDG activity could be noncalcified
FIGURE 3. Multifocal bone metastases: a 62-y-old patient with previous liver resection for HCC. ${ }^{18} \mathrm{~F}-\mathrm{FDG}$ and ${ }^{11} \mathrm{C}$ ACT PET/CT showed multifocal lung and bone metastases. Note that ${ }^{11} \mathrm{C}-\mathrm{ACT}$ revealed more bone metastasis than ${ }^{18} \mathrm{~F}-\mathrm{FDG}$ (e.g., right humeral and thoracic lesions [small arrows]), and ${ }^{11} \mathrm{C}-\mathrm{ACT}$ lesions are significantly more intense. Largest left iliac lesion (large arrows) showed typical osteolytic pattern on CT bone window.
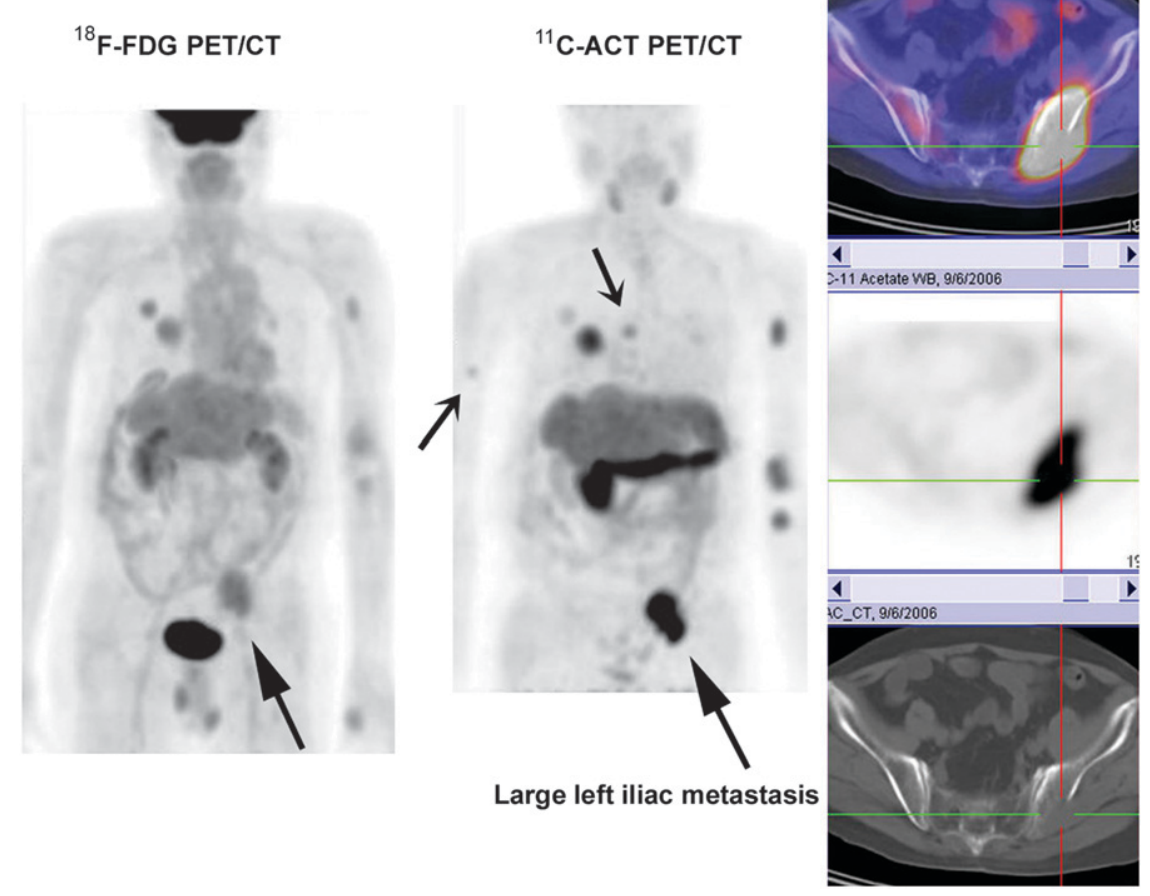
granulomatous nodes and, thus, be FP for metastasis. This possibility was minimized by correlation with serial PET/CT and other ancillary findings, such as concomitant biopsy-proven metastatic lung nodules (Fig. 2) and clinical follow-up.

The detection of bone metastasis showed no significant difference between ${ }^{18} \mathrm{~F}-\mathrm{FDG}$ and ${ }^{11} \mathrm{C}$-ACT (12 vs. 10 patients, respectively, in 15 patients with metastatic bone disease). However, unrevealed by the statistics, the metastatic bone lesions avid for both tracers were usually more avid for ${ }^{11} \mathrm{C}$-ACT than for ${ }^{18} \mathrm{~F}-\mathrm{FDG}$ (Fig. 3).

\section{Limitations}

As in many retrospective studies, the histopathologic confirmation of metastatic lesions is not always possible, particularly in patients with multiple sites or organs of involvement, multiple lesions, small lesions, bone lesions, or lesions in locations where biopsy is difficult or risky. Fortunately, most patients in our study had a high pretest probability of metastasis and many had serial imaging studies for comparison; thus, the likelihood of conflicting statistics was small. Another limitation of this study is the difficulty in statistical analysis on a patient basis, because a single patient could have multiple coexisting metastatic lesions with different tracer avidities. Therefore, the statistics on a lesion basis should be more reliable for practical purposes.

\section{CONCLUSION}

Accurate assessment of metastasis, staging, and therapy of HCC requires a precise imaging tool. The current study confirms that ${ }^{18} \mathrm{~F}-\mathrm{FDG}$ PET alone has a reasonable sensitivity in the detection of HCC metastasis, although it is not sensitive enough for the evaluation of HCC primary tumor. This study has focused on the PET/CT evaluation of metastatic $\mathrm{HCC}$ with a dual-tracer protocol $\left({ }^{11} \mathrm{C}\right.$-ACT and ${ }^{18} \mathrm{~F}-\mathrm{FDG}$ ), and our results suggest that it has a mutual complementary advantage, similar to the conclusion found previously in the detection of primary HCC tumors.

\section{ACKNOWLEDGMENTS}

The authors express their sincere gratitude to Dr. Shiu Man Hee of the Department of Surgery and Dr. Thomas
Leung of the Department of Oncology, Hong Kong Sanatorium and Hospital, for their valuable suggestions.

\section{REFERENCES}

1. Ho CL, Yu SC, Yeung DW. ${ }^{11} \mathrm{C}$-Acetate PET imaging in hepatocellular carcinoma and other liver masses. J Nucl Med. 2003;44:213-221.

2. Iwata Y, Shiomi S, Sasaki N, et al. Clinical usefulness of positron emission tomography with fluorine-18-fluorodeoxyglucose in the diagnosis of liver tumors. Ann Nucl Med. 2000;14:121-126.

3. Khan M, Combs C, Brunt E, et al. Positron emission tomography scanning in the evaluation of hepatocellular carcinoma. J Hepatol. 2000;32:792-797.

4. Bohm B, Voth M, Geoghegan J, et al. Impact of positron emission tomography on strategy in liver resection for primary and secondary liver tumors. $J$ Cancer Res Clin Oncol. 2004;130:266-272.

5. Chen YK, Hsieh DS, Liao CS, et al. Utility of FDG-PET for investigating unexplained serum AFP elevation in patients with suspected hepatocellular carcinoma recurrence. Anticancer Res. 2005;25:4719-4725.

6. Sugiyama M, Sakahara H, Torizuka T, et al. ${ }^{18}$ F-FDG PET in the detection of extrahepatic metastases from hepatocellular carcinoma. J Gastroenterol. 2004; 39:961-968.

7. Trojan J, Schroeder O, Raedle J, et al. Fluorine-18 FDG positron emission tomography for imaging of hepatocellular carcinoma. Am J Gastroenterol. 1999; 94:3314-3319.

8. Wudel LJ Jr, Delbeke D, Morris D, et al. The role of $\left[{ }^{18} \mathrm{~F}\right]$ fluorodeoxyglucose positron emission tomography imaging in the evaluation of hepatocellular carcinoma. Am Surg. 2003;69:117-124; discussion 124-116.

9. Ho CL, Cheng TKC, Yeung DWC. ${ }^{11} \mathrm{C}$ acetate and ${ }^{18} \mathrm{~F}$ FDG PET-CT imaging in hepatocellular carcinoma less than $2 \mathrm{~cm}$ [abstract]. J Nucl Med. 2005; 46(suppl):46P.

10. Bruix J, Sherman M, Llovet JM, et al. Clinical management of hepatocellular carcinoma: conclusions of the Barcelona-2000 EASL Conference-European Association for the Study of the Liver. J Hepatol. 2001;35:421-430.

11. Norenberg J, Simpson NR, Dunn BB, et al. Remote synthesis of $\left[{ }^{11} \mathrm{C}\right]$ acetate. Appl Radiat Isot. 1992;43:943-945.

12. Dodd GD 3rd, Baron RL, Oliver JH 3rd, Federle MP, Baumgartel PB. Enlarged abdominal lymph nodes in end-stage cirrhosis: CT-histopathologic correlation in 507 patients. Radiology. 1997;203:127-130.

13. Talwalkar JA, Gores GJ. Diagnosis and staging of hepatocellular carcinoma. Gastroenterology. 2004;127:S126-S132.

14. Katyal S, Oliver JH 3rd, Peterson MS, Ferris JV, Carr BS, Baron RL. Extrahepatic metastases of hepatocellular carcinoma. Radiology. 2000;216:698703.

15. Kim HC, Kim TK, Sung KB, et al. CT during hepatic arteriography and portography: an illustrative review. Radiographics. 2002;22:1041-1051.

16. Borghetti M, Benelli G, Bonardi R, Reduzzi L, Iori M. Bone metastasis of hepatocarcinoma: review of the literature, radiologic pictures and personal caseload [in Italian]. Radiol Med (Torino). 1991;82:48-51.

17. Kuhlman JE, Fishman EK, Leichner PK, Magid D, Order SE, Siegelman SS. Skeletal metastases from hepatoma: frequency, distribution, and radiographic features. Radiology. 1986;160:175-178.

18. Natsuizaka M, Omura T, Akaike T, et al. Clinical features of hepatocellular carcinoma with extrahepatic metastases. J Gastroenterol Hepatol. 2005;20: 1781-1787.

19. Efremidis SC, Vougiouklis N, Zafiriadou E, et al. Pathways of lymph node involvement in upper abdominal malignancies: evaluation with high-resolution CT. Eur Radiol. 1999;9:868-874. 